\title{
Strict diagonal dominance in asymptotic stability of general equilibrium
}

\author{
Sheng-Yi Hsu and Mau-Hsiang Shih*
}

Dedicated to Professor Wataru Takahashi on the occasion of his seventieth birthday

${ }^{*}$ Correspondence:

mhshih@math.ntnu.edu.tw

Department of Mathematics,

National Taiwan Normal University,

88 Sec. 4, Ting Chou Road, Taipei,

11677, Taiwan

\section{Springer}

\begin{abstract}
Stability of general equilibrium is usually depicted by a dynamic process of price adjustment which makes the flow of prices eventually come to rest at certain prices, so that the supply and demand of every commodity tend to equal each other. Here we construct a dynamical system of a competitive economy and find that the strict diagonal dominance of the Jacobian matrix of the excess demand function at its equilibrium guarantees the asymptotic stability.

MSC: 37N40; 91B55; 93D20
\end{abstract}

Keywords: fixed point; general equilibrium; stability; strict diagonal dominance

Economists look for conditions that make the equilibrium under consideration stable. For example, in [1], Arrow, Block and Hurwicz establish the stability on the assumption that the underlying excess demand function encoding the dynamics satisfies some gross substitute conditions; in [2], Uzawa also supposes that a gross substitute condition prevails and that the stability is guaranteed by using Lyapunov's stability theorem; in [3], Bear finds the equivalence of the stability of 'lagged' systems and 'first-order aggregation' of lagged systems. Our condition is that the excess demand function has a strictly diagonally dominant Jacobian matrix at its equilibrium. Conditions of this sort can also be found in $[4,5]$, where Hadar assumes the excess demand function to have a strictly diagonally dominant Jacobian matrix at not only a single point, but everywhere. By using a contraction technique, Hadar is able to show that the dynamical system has a unique equilibrium which is globally stable. In spite of the stronger conclusion, the assumption made in $[4,5]$ imposes too strong restriction on the excess demand function as stated by Hadar. Here we construct a dynamical system of a competitive economy and find that the strict diagonal dominance of the Jacobian matrix of the excess demand function at its equilibrium guarantees the asymptotic stability. Our analysis is based on the estimation of the range of eigenvalues and solving recurrences and concludes the local stability of the general equilibrium.

We begin with a mathematical formation of the relations between supply, demand, and prices in an economy. Consider an economy with commodities $1, \ldots, n$. Each commodity has its own price $p_{i} \in \mathbb{R}_{+}=\{x \in \mathbb{R} ; x \geq 0\}$. The vector $p=\left(p_{1}, \ldots, p_{n}\right)$ is called a price system. While we say vectors $d=\left(d_{1}, \ldots, d_{n}\right) \in \mathbb{R}_{+}^{n}$ and $s=\left(s_{1}, \ldots, s_{n}\right) \in \mathbb{R}_{+}^{n}$ are demand and supply, respectively, the components $d_{i}$ and $s_{i}$ represent, respectively, the demand and supply of commodity $i, i=1, \ldots, n$. A vector $z=\left(z_{1}, \ldots, z_{n}\right) \in \mathbb{R}^{n}$ is called an excess de-

(C) 2013 Hsu and Shih; licensee Springer. This is an Open Access article distributed under the terms of the Creative Commons Attribution License (http://creativecommons.org/licenses/by/2.0), which permits unrestricted use, distribution, and reproduction in any medium, provided the original work is properly cited. 
mand, while we think $z=d-s$ for some demand $d \in \mathbb{R}_{+}^{n}$ and some supply $s \in \mathbb{R}_{+}^{n}$. Suppose that a price system $p$ is given. Each consumer or producer in the economy will make consumption or produce commodities according to the price system $p$, resulting in a demand $D(p)=\left(d_{1}(p), \ldots, d_{n}(p)\right) \in \mathbb{R}_{+}^{n}$ and a supply $S(p)=\left(s_{1}(p), \ldots, s_{n}(p)\right) \in \mathbb{R}_{+}^{n}$ corresponding to $p$. The excess demand function is defined by $F=\left(f_{1}, \ldots, f_{n}\right)=D-S$. If there is a price system $p^{*} \in \mathbb{R}_{+}^{n}$ such that $F\left(p^{*}\right)=0$, meaning that the demand in each market is equal to the supply, then the state $\left(p^{*}, F\left(p^{*}\right)\right)=\left(p^{*}, 0\right)$ of prices and excess demand is called a general equilibrium of the economy.

The evolutionary process of the prices and excess demand is generated by the following method of price adjustment. Suppose that the economy has an excess demand $z \in \mathbb{R}^{n}$ and a price system $p \in \mathbb{R}_{+}^{n}$ at some time step. Then the price system at the next time step is given by

$$
p+L_{R}(z)
$$

where $R=\operatorname{diag}\left(r_{1}, \ldots, r_{n}\right)$ is an $n \times n$ diagonal matrix with $r_{i}>0$ for all $i=1, \ldots, n$, and $L_{R}(z)=\left(r_{1} z_{1}, \ldots, r_{n} z_{n}\right)$. It can be seen from a direct computation that the price of a certain commodity increases (decreases) if the demand of the commodity is larger (less) than its supply, which is sensible. From the computation we also see that each $r_{i}$ represents the scale of how much the price of commodity $i$ should be adjusted. Thus $r_{i}$ is considered as the adjustment speed of the price of commodity $i$. Now combining the excess demand function and the price adjustment gives the following discrete dynamical system of the economy:

$$
x(t+1)=\mathscr{F}(x(t)), \quad t=0,1,2, \ldots,
$$

where $\mathcal{F}: \mathbb{R}_{+}^{n} \times \mathbb{R}^{n} \rightarrow \mathbb{R}_{+}^{n} \times \mathbb{R}^{n}$ is defined by

$$
\mathcal{F}(p, z)=\left(p+L_{R}(z), F(p)\right), \quad p \in \mathbb{R}_{+}^{n}, z \in \mathbb{R}^{n} .
$$

In this dynamical system, a state variable $x \in \mathbb{R}_{+}^{n} \times \mathbb{R}^{n}$ represents a state of the economy with the first $n$ components of $x$ standing for the price system and the remaining ones standing for the excess demand.

Suppose $p^{*} \in \mathbb{R}_{+}^{n}, F\left(p^{*}\right)=0$, and $F$ is differentiable at $p^{*}$. Then $\left(p^{*}, 0\right)$ is a fixed point of $\mathcal{F}\left(i . e ., \mathcal{F}\left(p^{*}, 0\right)=\left(p^{*}, 0\right)\right)$ and is also a general equilibrium of the economy. Recall that the equilibrium $\left(p^{*}, 0\right)$ of system (1) is Lyapunov stable if all sufficiently small disturbances away from it damp out in time. Recall also that the equilibrium $\left(p^{*}, 0\right)$ of system $(1)$ is attracting if all trajectories $x(t)$ that start near $\left(p^{*}, 0\right)$ approach it as $t \rightarrow \infty$. Furthermore, the equilibrium $\left(p^{*}, 0\right)$ of system (1) is asymptotically stable if it is both Lyapunov stable and attracting. To show the stability of $\left(p^{*}, 0\right)$, we make the following assumptions:

(i) every component of $p^{*}$ is not zero;

(ii) the Jacobian matrix $\left(\frac{\partial f_{i}}{\partial p_{j}}\left(p^{*}\right)\right)$ is strictly diagonally dominant, i.e.,

$$
\sum_{j \neq i}\left|\frac{\partial f_{i}}{\partial p_{j}}\left(p^{*}\right)\right|<\left|\frac{\partial f_{i}}{\partial p_{i}}\left(p^{*}\right)\right|, \quad i=1, \ldots, n ;
$$

(iii) $\frac{\partial f_{i}}{\partial p_{i}}\left(p^{*}\right)<0$ for all $i=1, \ldots, n$. 
Assumption (i) means that none of the commodities has a zero equilibrium price. Assumption (ii) means that each 'own-good' price effect dominates the sum of the effects of the respective price on all other markets. Assumption (iii) means that a price raise (decrease) of some commodity should reduce (increase) its excess demand. As demonstrated by the following theorem, $\left(p^{*}, 0\right)$ will be asymptotically stable while the adjustment speeds of the prices lie in a suitable range.

Theorem 1 There is a $K>0$ such that the equilibrium $\left(p^{*}, 0\right)$ of system $(1)$ is asymptotically stable whenever $\max _{i} r_{i} \in(0, K)$.

To show this, let us introduce the following simple result.

Lemma 1 Suppose that $A$ is a $k \times k$ complex Jordan canonical form. If $A$ is invertible, then there exists a $k \times k$ upper triangular matrix $B$ such that $B^{2}=A$.

Proof Without lost of generality, we may assume that $A$ is a Jordan block with diagonals $c^{2}$ for some nonzero complex number $c$. Let $x_{1}=c, x_{2}=\frac{1}{2 x_{1}}$, and $x_{k}=-\frac{1}{2 x_{1}} \sum_{i=2}^{k-1} x_{i} x_{k-i+1}$ for all $k=3,4, \ldots, n$. Define $B=\left(b_{i j}\right)$, where

$$
b_{i j}= \begin{cases}0, & \text { if } i>j, \\ x_{j-i+1}, & \text { if } i \leq j .\end{cases}
$$

Then a computation shows that $B^{2}=A$.

For a square complex matrix $A$, the notation $\sigma(A)$ denotes the collection of all eigenvalues of $A$.

Proof of Theorem 1 Let us first consider the dynamical system

$$
y(t+1)=g(y(t)), \quad t=0,1,2, \ldots,
$$

where $g: \mathbb{R}_{+}^{2 n} \rightarrow \mathbb{R}_{+}^{2 n}$ is defined by

$$
g\left(p_{1}, p_{2}\right)=\left(p_{2}, p_{2}+L_{R}\left(F\left(p_{1}\right)\right)\right), \quad p_{1} \in \mathbb{R}_{+}^{n}, p_{2} \in \mathbb{R}_{+}^{n} .
$$

Then $\left(p^{*}, p^{*}\right)$ is an equilibrium of system (2) and the Jacobian matrix of $g$ at $\left(p^{*}, p^{*}\right)$ is

$$
M=\left(\begin{array}{cc}
O & I \\
R A & I
\end{array}\right),
$$

where $I, O$, and $A=\left(a_{i j}\right)$ are the $n \times n$ identity matrix, the zero matrix, and the Jacobian matrix $\left(\frac{\partial f_{i}}{\partial p_{j}}\left(p^{*}\right)\right)$, respectively. Since $a_{i i}=\frac{\partial f_{i}}{\partial p_{i}}\left(p^{*}\right)<0$ and $r_{i}>0$ for all $i=1, \ldots, n$, there is a positive number $K$ such that if $\max _{i} r_{i}<K$, then

$$
-\frac{1}{8}<r_{i} a_{i i}<0, \quad i=1, \ldots, n .
$$


Now suppose $\max _{i} r_{i} \in(0, K)$. Let $c=\min _{i} r_{i} a_{i i} \in\left(-\frac{1}{8}, 0\right)$. If $\left|z-r_{i} a_{i i}\right|<\left|r_{i} a_{i i}\right|$ for some $i=$ $1, \ldots, n$, then

$$
|z-c| \leq\left|z-r_{i} a_{i i}\right|+\left|r_{i} a_{i i}-c\right|<\left|r_{i} a_{i i}\right|+r_{i} a_{i i}-c=-c
$$

Since $A=\left(\frac{\partial f_{i}}{\partial p_{j}}\left(p^{*}\right)\right)$ is strictly diagonally dominant, so is $R A$. Hence, by Gersgorin theorem [6] and (3), for any eigenvalue $\alpha$ of $R A$ we have

$$
\alpha \in \bigcup_{i=1}^{n}\left\{z \in \mathbb{C} ;\left|z-r_{i} a_{i i}\right|<\left|r_{i} a_{i i}\right|\right\} \subset\{z \in \mathbb{C} ;|z-c|<-c\}
$$

Suppose $\alpha=r(\cos \theta+i \sin \theta)+c$, where $r \geq 0$ and $0<\theta \leq 2 \pi$. Then by (4) we have

$$
r=|r(\cos \theta+i \sin \theta)|=|r(\cos \theta+i \sin \theta)+c-c|=|\alpha-c|<-c
$$

Hence $c<r \cos \theta<-c$. Now $-\frac{1}{8}<c<0$ implies $-\frac{1}{4}<r \cos \theta+c<0$, i.e.,

$$
-\frac{1}{4}<\operatorname{Re}(\alpha)<0, \quad \alpha \in \sigma(R A)
$$

where $\operatorname{Re}(\alpha)$ denotes the real part of $\alpha$. Suppose $J$ is a Jordan canonical form of $R A$. Then by (5) we have

$$
0<\operatorname{Re}(\alpha)<\frac{1}{4}, \quad \alpha \in \sigma\left(J+\frac{1}{4} I\right)
$$

Therefore, $\alpha \neq 0$ for all $\alpha \in \sigma\left(J+\frac{1}{4} I\right)$, and hence there is an $n \times n$ upper triangular matrix $B$ such that $B^{2}=J+\frac{1}{4} I$ by the lemma. For any $\beta \in \sigma(B)$,

$$
\beta^{2}-\frac{1}{4} \in \sigma(J)=\sigma(R A)
$$

Thus by (4) we have

$$
\left|\beta^{2}-\frac{1}{4}-c\right|<-c
$$

Therefore,

$$
\begin{aligned}
|\beta|^{2} & =\left|\beta^{2}\right|=\left|\beta^{2}-\left(\frac{1}{4}+c\right)+\left(\frac{1}{4}+c\right)\right| \\
& \leq\left|\beta^{2}-\left(\frac{1}{4}+c\right)\right|+\left|\frac{1}{4}+c\right| \\
& <-c+\frac{1}{4}+c=\frac{1}{4} .
\end{aligned}
$$

Thus $|\beta|<\frac{1}{2}$ and hence

$$
\left|\frac{1}{2} \pm \beta\right| \leq \frac{1}{2}+|\beta|<1, \quad \beta \in \sigma(B) .
$$


Since $B$ is upper triangular, it follows that all the eigenvalues of matrices $\frac{1}{2} I \pm B$ lie in the open unit disc. For $k=0,1,2, \ldots$, put

$$
\begin{aligned}
& N_{1}(k)=\left(\frac{1}{2} B-\frac{1}{8} B^{-1}\right)\left(\frac{1}{2} I+B\right)^{k}-\left(\frac{1}{2} B-\frac{1}{8} B^{-1}\right)\left(\frac{1}{2} I-B\right)^{k}, \\
& N_{2}(k)=\left(\frac{1}{2} I+\frac{1}{4} B^{-1}\right)\left(\frac{1}{2} I+B\right)^{k}+\left(\frac{1}{2} I-\frac{1}{4} B^{-1}\right)\left(\frac{1}{2} I-B\right)^{k} .
\end{aligned}
$$

Then by (6) we conclude that

$$
\lim _{k \rightarrow \infty} N_{1}(k)=\lim _{k \rightarrow \infty} N_{2}(k)=O .
$$

Since $R A$ is similar to $J$, the Jacobian matrix $M$ of $g$ at $\left(p^{*}, p^{*}\right)$ is similar to the matrix

$$
N=\left(\begin{array}{ll}
O & I \\
J & I
\end{array}\right) .
$$

We claim that for $k=0,1,2, \ldots$,

$$
N^{k}=\left(\begin{array}{cc}
N_{1}(k-1) & N_{2}(k-1) \\
N_{1}(k) & N_{2}(k)
\end{array}\right) .
$$

We shall proceed by induction. When $k=0$, (8) follows from a direct computation. Now suppose (8) holds for $k=l$. Then

$$
\begin{aligned}
N^{l+1} & =N^{l} N=\left(\begin{array}{cc}
N_{1}(l-1) & N_{2}(l-1) \\
N_{1}(l) & N_{2}(l)
\end{array}\right)\left(\begin{array}{cc}
O & I \\
J & I
\end{array}\right) \\
& =\left(\begin{array}{cc}
N_{2}(l-1) J & N_{1}(l-1)+N_{2}(l-1) \\
N_{2}(l) J & N_{1}(l)+N_{2}(l)
\end{array}\right) .
\end{aligned}
$$

Note that the identity $J=\left(B+\frac{1}{2} I\right)\left(B-\frac{1}{2} I\right)$ shows that

$$
N_{2}(t-1) J=N_{1}(t), \quad t=1,2, \ldots
$$

Note also that

$$
N_{1}(t-1)+N_{2}(t-1)=N_{2}(t), \quad t=1,2, \ldots
$$

Hence (8) holds for $k=l+1$, completing the induction and proving the claim.

Since $M$ is similar to $N,(8)$ and (7) imply that $M^{k}$ goes to the zero matrix as $k$ goes to infinity, showing that every eigenvalue of $M$ lies in the open unit disc, so that the equilibrium $\left(p^{*}, p^{*}\right)$ of system (2) is stable and attracting.

Next we claim that the equilibrium $\left(p^{*}, 0\right)$ of system $(1)$ is asymptotically stable. The notations $x(t)$ and $y(t)$ will denote the flow of system (1) and (2), respectively. The norm $\|\cdot\|$ will stand for the supremum norm on $\mathbb{R}^{n}$. Let $U$ be an open neighborhood of $\left(p^{*}, 0\right)$. 
Since every component of $p^{*}$ is not zero, there is a $d>0$ such that $p \in \mathbb{R}_{+}^{n}$ if $\left\|p-p^{*}\right\|<d$. Without loss of generality, we may assume that $U=U_{1} \times U_{2}$, where

$$
\begin{aligned}
& U_{1}=\left\{p \in \mathbb{R}^{n} ;\left\|p-p^{*}\right\|<d\right\} \text { and } \\
& U_{2}=\left\{z \in \mathbb{R}^{n} ;\|z\|<d\right\} .
\end{aligned}
$$

By the continuity of $F$ at $p^{*}$, there is a $\delta \in(0, d)$ such that

$$
\|F(p)\|<d \quad \text { if }\left\|p-p^{*}\right\|<\delta .
$$

Suppose $y(t)=\left(y_{1}(t), y_{2}(t)\right)$, where $y_{1}(t) \in \mathbb{R}_{+}^{n}$ and $y_{2}(t) \in \mathbb{R}_{+}^{n}$ for all $t=0,1,2, \ldots$. By the asymptotical stability of the equilibrium $\left(p^{*}, p^{*}\right)$ of system $(2)$, there is a $\delta^{\prime} \in(0, \delta)$ such that if $\left\|y_{1}(0)-p^{*}\right\|<\delta^{\prime}$ and $\left\|y_{2}(0)-p^{*}\right\|<\delta^{\prime}$, then

$$
\begin{aligned}
& \left\|y_{1}(t)-p^{*}\right\|<\delta \text { and }\left\|y_{2}(t)-p^{*}\right\|<\delta, \quad t=1,2, \ldots, \text { and } \\
& y(t) \rightarrow\left(p^{*}, p^{*}\right) \quad \text { as } t \rightarrow \infty .
\end{aligned}
$$

Suppose $x(t)=(p(t), z(t))$, where $p(t) \in \mathbb{R}_{+}^{n}$ and $z(t) \in \mathbb{R}^{n}$ for all $t=0,1,2, \ldots$. Since $p(1)=$ $p(0)+L_{R}(z(0))$, there is an $\varepsilon \in\left(0, \delta^{\prime}\right)$ such that

$$
\left\|p(1)-p^{*}\right\|<\delta^{\prime} \quad \text { if }\left\|p(0)-p^{*}\right\|<\varepsilon \text { and }\|z(0)\|<\varepsilon .
$$

Now choose $x(0) \in \mathbb{R}_{+}^{n} \times \mathbb{R}^{n}$ so that $\left\|p(0)-p^{*}\right\|<\varepsilon$ and $\|z(0)\|<\varepsilon$. Put $y_{1}(0)=p(0)$ and $y_{2}(0)=p(1)$. Then a computation shows that

$$
y(t)=\left(y_{1}(t), y_{2}(t)\right)=(p(t), p(t+1)), \quad t=0,1,2, \ldots
$$

Moreover, by (12) we have

$$
\left\|y_{2}(0)-p^{*}\right\|=\left\|p(1)-p^{*}\right\|<\delta^{\prime} .
$$

Since $\left\|y_{1}(0)-p^{*}\right\|=\left\|p(0)-p^{*}\right\|<\varepsilon<\delta^{\prime}<\delta$, (10) implies

$$
\left\|p(t)-p^{*}\right\|=\left\|y_{1}(t)-p^{*}\right\|<\delta<d, \quad t=0,1,2, \ldots
$$

Thus (9) gives

$$
\|z(t)\|=\|F(p(t-1))\|<d, \quad t=1,2, \ldots
$$

Therefore,

$$
x(t)=(p(t), z(t)) \in U_{1} \times U_{2}=U, \quad t=0,1,2 \ldots,
$$

meaning that the equilibrium $\left(p^{*}, 0\right)$ of system (1) is stable. By the continuity of $F$ at $p^{*}$, (11) shows that $x(t) \rightarrow\left(p^{*}, 0\right)$ as $t \rightarrow \infty$. This completes the proof. 
Competing interests

The authors declare that they have no competing interests.

\section{Authors' contributions}

Both authors contributed equally and significantly in writing this paper. Both authors read and approved the final manuscript.

\section{Acknowledgements}

This work was supported by the National Science Council of the Republic of China.

Received: 14 June 2013 Accepted: 8 August 2013 Published: 28 August 2013

\section{References}

1. Arrow, KJ, Block, HD, Hurwicz, L: On the stability of the competitive equilibrium II. Econometrica 27, $82-109$ (1959)

2. Uzawa, H: The stability of dynamic processes. Econometrica 29, 617-631 (1961)

3. Bear, DVT: A matrix multiplier and distributed lags. Econometrica 31, 514-529 (1963)

4. Hadar, J: A note on dominant diagonals in stability analysis. Econometrica 33, 442-444 (1965)

5. Hadar, J: Dominant diagonals-a correction. Econometrica 37, 541-543 (1969)

6. Horn, RA, Johnson, CR: Matrix Analysis. Cambridge University Press, Cambridge (1985)

doi:10.1186/1687-1812-2013-225

Cite this article as: $\mathrm{Hsu}$ and Shih: Strict diagonal dominance in asymptotic stability of general equilibrium. Fixed Point Theory and Applications 2013 2013:225.

\section{Submit your manuscript to a SpringerOpen ${ }^{\circ}$ journal and benefit from:}

- Convenient online submission

- Rigorous peer review

- Immediate publication on acceptance

- Open access: articles freely available online

- High visibility within the field

- Retaining the copyright to your article 